\title{
APPLICATION CASE: PROTOTYPE OF RADAR TOWER STRUCTURAL HEALTH MONITORING SYSTEM
}

\author{
Deniss Mironovs ${ }^{1}$, Aleksey Mironov $^{2}$, Andris Chate ${ }^{1}$ \\ ${ }^{1}$ Institute of Materials and Structures, Riga Technical University, Latvia; \\ ${ }^{2}$ SIA "Vibroakustikas laboratorija", Latvia \\ info@rtu.lv,info@viblab.lv
}

\begin{abstract}
Industrial and civil structures like bridges, wind turbines, pipelines etc., are widely present in rural area. To maintain functionality of these structures, their condition is controlled using Structural Health Monitoring (SHM). SHM assists engineers in finding early structural defects like cracks, deformations, material deterioration, metal fatigue and so on. Monitoring can prevent accidents from happening by giving early warnings and a chance to perform necessary repairs and adjustments to the structure. It is efficient and economically reasonable to have possibility to perform planned repairs well ahead, instead of doing urgent fixing. What is more important, structural condition monitoring improves safety along with reducing maintenance costs. There are numerous non-destructive techniques (ultrasound, Fiber Bragg Grating, acoustic emission) applied for SHM, but the focus of this paper is on vibration-based methods, particularly - Operational Modal Analysis (OMA). The aim of the study is to validate SHM application possibility for a full scale industrial structure using the Operational Modal Analysis based SHM system. The system is designed as a cost-effective solution, mainly by using cheap piezo film deformation sensors and utilizing multi-patch OMA technique. A test was performed and modal parameters of the radar tower were obtained and compared with the FE model. The possibility to perform SHM on an industrial structure was validated.
\end{abstract}

Keywords: structural health monitoring, operational modal analysis, vibration, piezo film sensors.

\section{Introduction}

Structural Health Monitoring helps engineers control the condition of structures like wind turbines, bridges and others present in rural area. This is important for establishing safe and reliable operation of these structures [1]. What more, based on SHM given information, engineers can plan repairs well ahead, which ultimately saves operational costs. SHM techniques are based on different physical phenomena - vibration [2], ultrasound [3], acoustic emission [4] and strain [5], to name a few.

Some SHM systems require full stop of the structures operation (e.g., inspection of wind turbine blades) in order to perform SHM, which leads to serious operational expenses. Other systems, like Fibre Bragg Grating technology [5] based systems, are limited in frequency range, which makes it impossible to detect certain types of damages. Newly improved systems can overcome this obstacle, but these systems are expensive, mostly due to complicated but high quality equipment [6]. From this point of view, vibration based methods have considerable potential for further development [7], especially those methods that utilize modal analysis [8].

One of the vibration based system identification techniques is Operational Modal Analysis (OMA) [9];[10]. This approach has proven to be liable for structural health monitoring in [11], which focused on applying OMA based SHM to a scaled laboratory model of a wind turbine. The goal was to develop SHM system, that could be both efficient, cost-friendly and which would work while the structure is in operation. The presented work is a continuation of the study in [11].

The goal of this study is to validate SHM application possibility for a full scale industrial structure using the Operational Modal Analysis based SHM system. Based on that the tasks set out for this study are:

- design cost-efficient OMA measurement system prototype for selected test object.

- make sure that the used methods provide correct objects state identification. This is to be accomplished by validating experimental data with modelling data.

\section{Materials and methods}

This chapter focuses on methods and materials used for SHM system, including sensors. 


\subsection{Operational Modal Analysis}

The basis of the presented technology is Operational Modal Analysis. The aim of any modal analysis is to identify the systems modal parameters - frequency $f$, oscillationshape $\varphi$ and damping $\xi$, which describe the systems dynamic behaviour, i.e. how it oscillates and vibrates in response to excitation. Unlikewith classical modal analysis, where excitation is artificial, controlled and measured, OMA uses direct time responses of vibration signals from multiple sensors on a structure under natural excitation - wind, traffic, water flow and others. The main requirements for the excitation force in OMA are the following:

- The input forces are random in time, broadband and flat in frequency, and smooth. They are also assumed to be mutually uncorrelated,

- The forces are evenly distributed spatially on the system.

These requirements are satisfied, for instance, in a pipeline case (fluid flow), but are not fully met for the radar tower or wind turbine, due to extra rotational periodic load from the antenna and fan correspondingly. Nevertheless, excitation periodicity can be extracted, if needed [11]. Also, many structures have periodic excitation, so it is valuable to attempt the OMA approach with this kind of excitation.

By performing modal analysis and receiving information on the structures dynamic behaviour it is possible to draw conclusions about its condition. Modal analysis is a widely spread approach on the design stage of a structure. It is possible, however, to employ the modal analysis for SHM. For that one would need to perform OMA on a structure on a regular basis and compare the resulting modal parameters. For a healthy state of the structure, modal parameters should not change, and vice versa for a modified or faulty state, the structures modal parameters will change. By analysing frequency and shape modifications an experienced engineer can make conclusions on the type of modification local stiffness degradation, change of boundary conditions, etc.

\subsection{Multi-patch OMA}

Operational Modal Analysis requires multiple sensors installed on the structure, thus the same amount of input channels on the data acquisition unit (DAU) is required. It is possible to significantly reduce the cost of an SHM system, if one uses multi-patch OMA. In this OMA approach vibration is measured consequently by dividing sensors in small groups called patches at a time. Each group includes two types of channels: reference channels, which are not moved, and moving or roving channels. After all patches are measured, signal processing algorithms combine those patches together for further modal parameter estimation. Reference channels are used here to equalize the excitation energy that varies in time for different patches. With multi-patch OMA one can measure, say, 40 signals, with only 8 input channels. Of course, the time for data recording becomes longer, but the cost of the system is reduced significantly.

\subsection{Piezo Film sensors}

Another significant part of the SHM system price is the sensors. Industry standard accelerometer for vibrational signal measurement can cost hundreds of euros. For a multiple channels system for an industrial object this is quite expensive. An alternative is to use piezo film deformation sensors, which cost around EUR 10 per piece. These films provide wide frequency and dynamic range, and are well suited for vibration measurements. Transducer includes a sensor (piezo film), connector and preamplifier (Fig.1). Piezo film can also be used without preamplifier, but the sensitivity and output voltage are significantly lower.

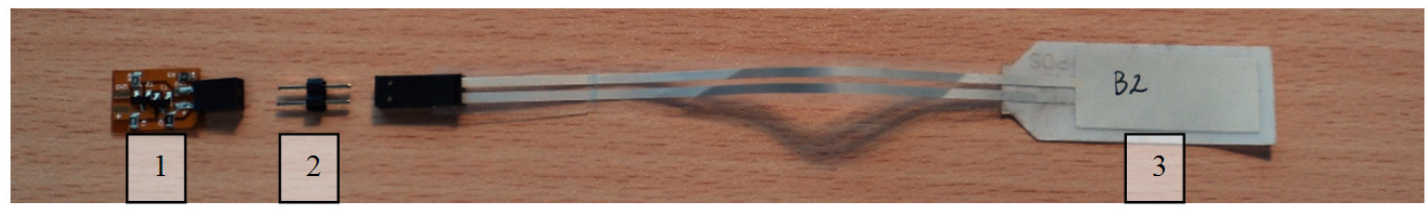

Fig.1. Deformation sensor: 1 - preamplifier; 2 - connector pins; 3 - piezo film with adhesive

Piezo film is a voltage source in series with a capacitor [12]. It has a thin piezo material between two layers of conducting silver plates. The film is flexible and is glued on to a surface. When the 
surface is deformed, film is also deformed, which results in an alternating voltage signal that mimics deformation oscillations. Due to the capacitor working principle, the output voltage signal is directly proportional to the velocity of deformation.

\subsection{Test object}

The structure under test is a radar tower in Riga airport (Fig. 2). It is $24 \mathrm{~m} \mathrm{high,} \mathrm{with} \mathrm{a} \mathrm{rotating}$ antenna on the top. The platform with the antenna is not supposed to be monitored, so the monitoring area is decided to be from the bottom of the tower to the fifth red frame section, which is $18 \mathrm{~m}$ high. There are 20 measurement points on the tower placed in corners of 5 horizontal sections (Fig. 2c). Fig. $2 \mathrm{~b}$ shows the middle of the section corner, where sensors are installed. The placement is chosen on each side of the corner, to register deformation in both directions. There are two sensors on each side, one is the main sensor, another is a reserve, in case the first one fails. There are 80 sensors on the tower, from which 40 can be used for simultaneous OMA measurements.

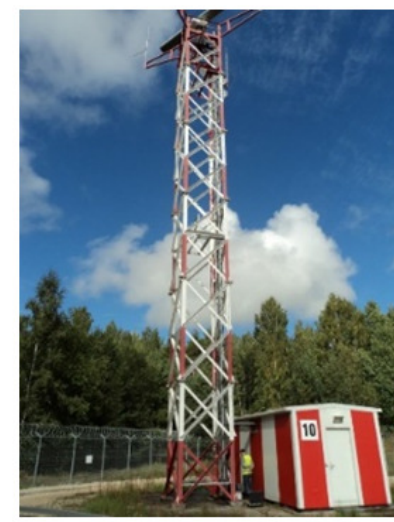

a)

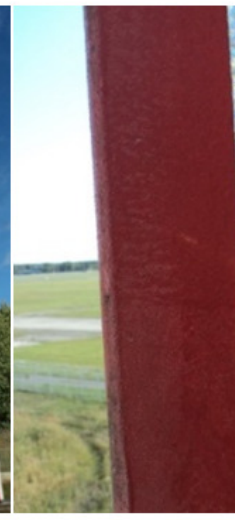

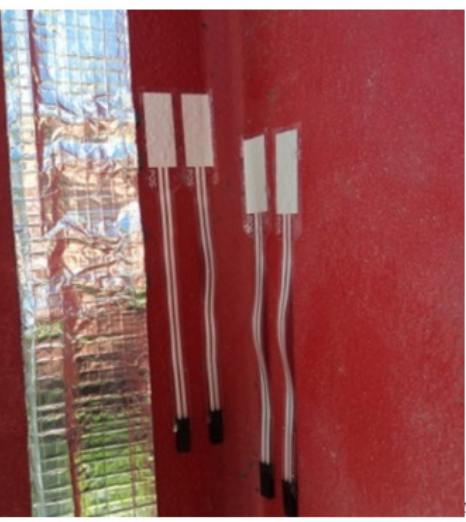

b)

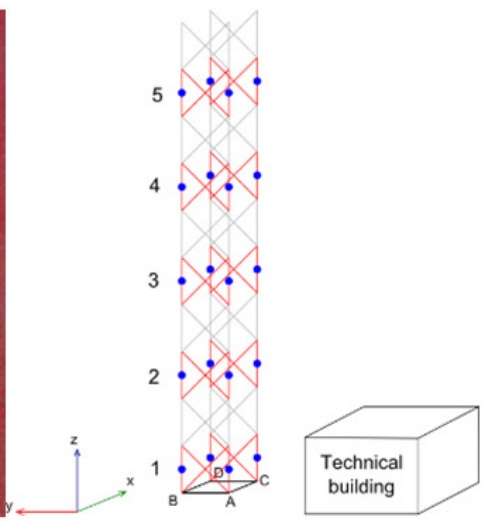

c)

Fig. 2. a) Radar tower; b) single measurement point; c) point locations (blue dots)

\subsection{Measurement cables and patching panel}

Measurement cables connect deformation sensors to DAU inputs. Each sensor requires 2 wires signal and floating ground. The cables used are flat ribbon cables (FRC). FRC cables are installed on each towers leg along its length. At designated places FRC wires are soldered to the preamplifiers placed next to the sensor positions. FRC with preamplifiers on both sides are covered with aluminium fibre reinforced shielding tape that protects wiring from electromagnetic interference (EMI) noise. The shield is grounded together with DAU and PC. When cables are installed properly, piezo films are connected to the preamplifiers. The ends of the FRC cables are terminated by D-sub connectors, which are connected to the patching panel (Fig. 3a). The panel itself has 80 SMB outputs, one for every existing sensor. This simple approach allows easily changing patching cables between sensors, if one fails or for multi-patch OMA purposes. Patching cables are then connected into designated input channels on the DAU (Fig. 3b).

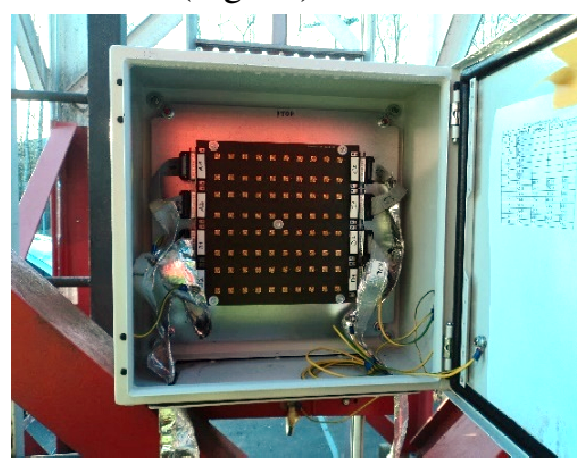

a)

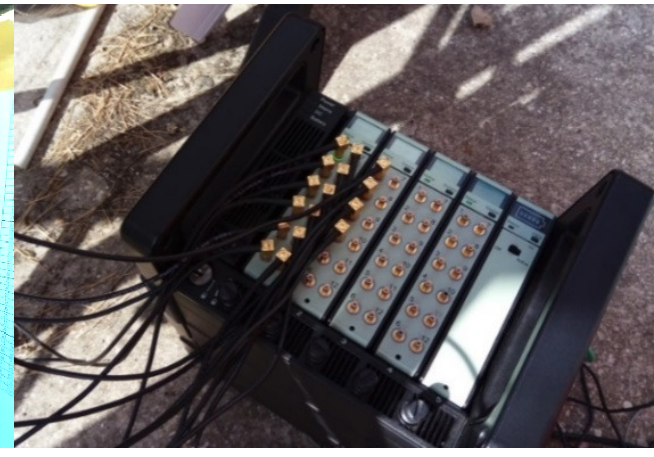

b)

Fig. 3. a) Patching panel; b) data acquisition unit 


\subsection{Installation}

The process of installation requires the weather conditions to be dry and not too windy. There are 4 shielded FRC cables, one for each tower side. The cables have double sided adhesive tape on one side. Using mobile lifter, engineers go up to the height of $18 \mathrm{~m}$, close to the $5^{\text {th }}$ level, with the cables and sensors ready. First, the tower surface is cleansed with cleaning solution. Then engineers start to glue cables and sensors at designated locations. When sensors are glued on the surface and connected to the preamplifier inputs, the remaining open part of the sensors is covered with aluminium adhesive tape, which serves as protection from EMI and bad weather conditions. This process continues for every level on each side. After all cables and sensors are installed, the ends of the cables are connected to D-sub connectors on the patching panel (Fig. 3a).The principal schematic of the measurement system is shown in Fig. 4.

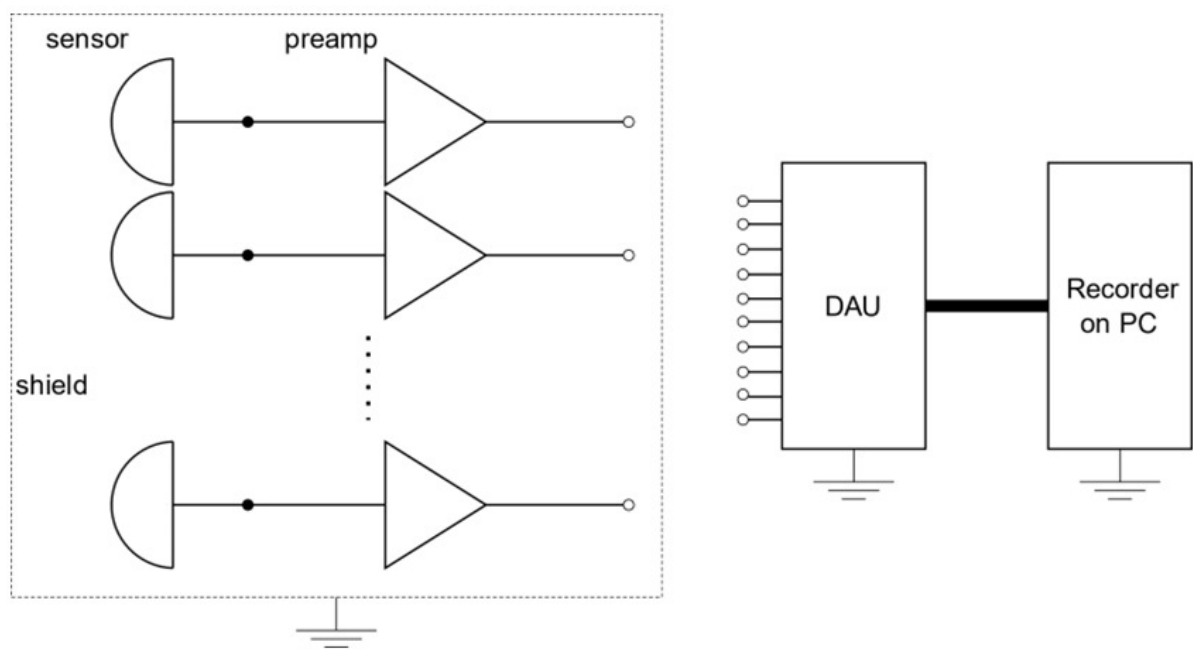

Fig. 4. Measurement system principal schematic

\section{Results and discussion}

This section covers the obtained analytical and experimental results with discussion for the monitoring process.

\subsection{Multi-patch OMA measurements}

After the system, which included sensors, cables, patching panel and other necessary equipment, was installed, multi-patch OMA measurement session was performed. Weather conditions play a significant role for these tests - it should be windy enough, as wind is the main random excitation source. The wind speed during the experiment was slow, thus the main excitation source was periodic - rotating antenna.

The measurement setup was the following. One patch consisted of 16 channels, 8 of which are reference channels, thus another 8 are moving channels. The full session takes 4 measurements, 5 minutes each. Between the measurement the patching cables were reattached to the next measured channels.

\subsection{Finite element analysis}

Finite element (FE) analysis based on NeiNastran software package was done for a digital tower model for the purpose of obtaining analytical modal parameters. Some of modal frequencies are shown in Table 2. Based on these results the frequency range for measurement recordings was defined to be $200 \mathrm{~Hz}$, thus the sampling frequency is $512 \mathrm{~Hz}$. Some modes have identical shapes, but in different directions. For example, Modes 1 and 2 are both $1^{\text {st }}$ bending shapes, but in mutually perpendicular axes. 


\subsection{Experimental results}

Vibration recordings were processed using Svibs Artemis software for OMA. Artemis performs estimation of modal parameters by building mathematical models that fit the experimental data. Some of the approaches can be found in [10]. The result is a set of experimental modal parameters.

Fig. 5 shows modal shapes for the $1^{\text {st }}$ bending mode. In Fig. $5 \mathrm{a}$ one can see analytical $1^{\text {st }}$ bending shape in $\mathrm{Y}$ direction, Fig. $5 \mathrm{~b}$ is the tower geometry in steady state for comparison, and Fig. 5c shows experimental $1^{\text {st }}$ bending mode shape. The latter is a combined shape for bending shapes in $\mathrm{X}$ and $\mathrm{Y}$ direction. The shape is estimated as combined, because the frequency resolution in OMA estimation is not fine enough to be able to separate two closely spaced modes. Also, due to periodic antenna excitation, the tower oscillation shape has torsional movement around its axis. This is observed for most shapes, not only the $1^{\text {st }}$ bending one. Note, that experimental shapes show local deformation caused by stress, which is inversely proportional to displacement in the analytical results. Due to this it is impractical to quantitatively compare the analytical and experimental shapes, but it is possible to analyse similarities and differences visually or in reference domain.

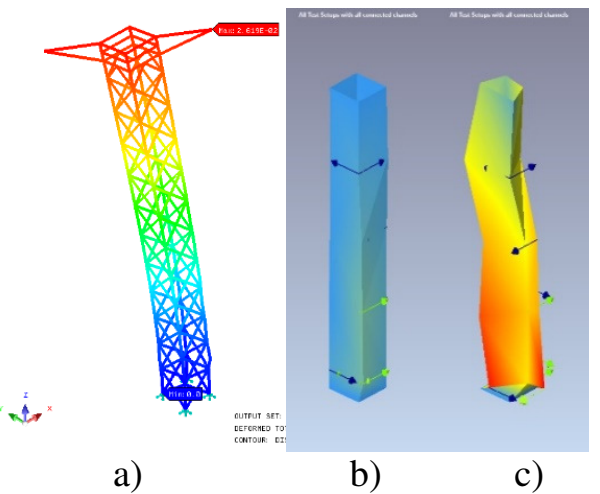

Fig. 5. a) Analytical $1^{\text {st }}$ bending; b) Experimental steady state; c) Experimental $1^{\text {st }}$ bending.

Table 1 shows experimental modal frequencies. The first experimental mode correlates well with the analytical one $(2.21 \mathrm{~Hz}$ and $2.14 \mathrm{~Hz}$ correspondingly) (Table 2). The first mode correlation between FE and test data is a good indication that the FE model is adequate. However, to be more confident, it is necessary to compare other modes as well, using the Modal Assurance Criterion, for example. In order to do this, FE model displacements should be transformed into deformations. This is a subject of future research.

Table 1

Experimental modal frequencies

\begin{tabular}{|c|c|c|c|c|c|c|c|c|}
\hline $\mathrm{Nr}$. & 1 & 2 & 3 & 4 & 5 & 6 & 7 & 8 \\
\hline$f, \mathrm{~Hz}$ & 2.21 & 5.75 & 9.16 & 12.84 & 13.99 & 16.93 & 59.24 & 76.88 \\
\hline
\end{tabular}

Table 2 shows comparison of modal frequencies between the experimental and analytical results. It is observed, that the mode order is different for the analytical result. Analytical console modes do not create significant vibrational response in the tower, so these are not identified. Other differences can also be explained by the fact that frame sections in the technical project were $4 \mathrm{~m}$ high. The measurement of the real section showed $3.6 \mathrm{~m}$. Full measurement of the real tower dimensions was not feasible, so the difference between the model and the object remained.

Table 2

Comparison of modal frequencies

\begin{tabular}{|l|l|l|l|l|}
\hline \multicolumn{3}{|c|}{ Analytical } & \multicolumn{2}{c|}{ Experimental } \\
\hline Mode Nr. & f, Hz & Shape & Mode Nr. & f, Hz \\
\hline 1 & 1.92 & $1^{\text {st }}$ bending & \multirow{2}{*}{1} & 2.21 \\
\hline 2 & 2.14 & pair & & \multirow{2}{*}{12.84} \\
\hline 5 & 12.11 & $2^{\text {nd }}$ bending & \multirow{2}{*}{4} & 16.93 \\
\hline 6 & 12.76 & pair & & 6 \\
\hline 8 & 16.47 & $1^{\text {st }}$ torsional & 6 & \multicolumn{2}{|l}{} \\
\hline
\end{tabular}




\subsection{Monitoring prospects}

Modal frequencies (Table ) and shapes describe dynamic behaviour of the tower in its current state or condition. Once the condition of the tower is modified (local corrosion, loose bolts, base problem caused by earth erosion, added mass etc.), the dynamic behaviour changes and the modal parameters also change. By monitoring the modal parameters it is possible to detect the condition modification. By analysing how modal parameters changed it is then also possible to identify and localize defects.

In [11] two methods were presented that make estimation of the condition change based on the modal parameters. These methods are called Modal Parameter Variation Intensity (MPVI) and Singular Value Change Assessment (SVCA). The first one is very useful for defect identification and localization, but requires to perform modal parameter estimation, which is usually a manual process. The second method can only detect condition modification and does not provide any insight about the type of the defect or its localization. However, SVCA does not require modal parameter estimation, which allows to create an automatic algorithm for structural health monitoring.

\subsection{Discussion on experimental SHM system in industrial environment.}

The developed experimental measurement system has turned out to be cost-efficient, as the used materials are widely accessible and inexpensive. Multi-patch OMA approach has proven to be applicable using the measurement system. The shielding was efficient and EMI related noise was largely masked by the vibration signal.

One month after the experiment the system was checked and OMA measurements were repeated. To the authors regret, some of the system channel signals were corrupted. There was either low and noisy signal transmission, or no vibrational signal at all. Measurement network troubleshooting was done, which did not reveal connectivity problems. It is believed that environmental conditions had a strong impact on transducers preamplifiers. Two particular causes are

- humidity can create short circuit in the preamplifier,

- strong electromagnetic field could damage field-effect transistors (FET) in the preamplifier.

Condensed water was observed around the preamplifier after opening that area, which means that the cable was not sealed well enough. In laboratory conditions it was also observed that preamplifiers sometimes failed after touching them with bare hands, which could lead to static voltage discharge and FET damage.

It is obvious, that the weakest part of the prototype is the preamplifier. The authors consider to either improve the circuitry with more reliable components or develop insulation solution with at least IP65 rating.

\section{Conclusions}

The goal of the study was to validate SHM application possibility for a full scale industrial structure using the Operational Modal Analysis based SHM system. For that a cost-efficient OMA measurement system prototype for $20 \mathrm{~m}$ airport radar tower was designed. Cost-efficiency was gained by utilizing inexpensive deformation piezo film sensors and by reducing the amount of input channels in the measurement system by switching to multi-patching.

The design and installation process were done. Measurement procedures were developed and applied. The resulting modal parameters provide enough description of the tower dynamic behaviour. Modal parameters can be later used for monitoring purposes.

There is a plan to continue work on developing the structural health monitoring system. This includes reliability improvement, mostly through a careful circuitry design. Another direction is improvement of signal processing algorithms. Lastly, attention will be given to automatization of the SHM system by utilizing multiplexing and automatic signal processing.

\section{Acknowledgement}

The paper uses materials related to research study No. 1.7 "Research and Development of Experimental Technology of Technical Condition Monitoring of Industrial Structures" of the project 
„Establishment of Mechanical Engineering Competence Centre” in cooperation with Central Finance and Contracting Agency of Latvia (1.2.1.1/16/A/003).

\section{References}

[1] Fritzen C.P., Güemes A., Balageas D. Structural Health Monitoring. ISTE, 2006. 13-44 pp.

[2] Doebling S.W., Farrar C.R., Prime M.B. A summary review of vibration-based damage identification methods, The Shock and Vibration Digest, Vol. 30, pp. 91-105, 1998.

[3] Aranguren G., Monje P.M., Cokonaj V., et al. Ultrasonic wave-based structural health monitoring embedded instrument. Review of Scientific Instruments, Vol. 84, Issue 12, 2013.

[4] Smithard J., Rajic N., van der Velden S., et al.An Advanced Multi-Sensor Acousto-Ultrasonic Structural Health Monitoring System: Development and Aerospace Demonstration. Proceedings of 6th Asia Pacific Workshop on Structural Health Monitoring. Procedia Engineering 188, 2017, 448-455 pp.

[5] Strong A., Sanderson N., Lees G., et al. A comprehensive distributed pipeline condition monitoring system and its field trial. Proceedings of IPC'08, Calgary, September 29-October 3, 2008.

[6] HartogA.H., LiokumovichL. B., UshakovN., ConstantinouA. The Use of Multi-frequency Acquisition to Significantly Improve the Quality of Fibre-optic Distributed Vibration Sensing. 78th EAGE Conference and Exhibition, May 2016.

[7] Randall R.B. Vibration-based Condition Monitoring. John Wiley \& Sons, 2011.

[8] Kovalovs A., Rucevskis S., Akishin P., et al. Numerical Investigation on Detection of Prestress Losses in a Prestressed Concrete Slab by Modal Analysis. IOP Conf. Series: Materials Science and Engineering, Vol 251, 2017.

[9] Brincker R., Ventura C., Introduction to Operational Modal Analysis. Wiley, 2015

[10]Zhang L., Brincker R., Andersen P. An Overview of Operational Modal Analysis: Major Development and Issues. Proc. 1st International Operational Modal Analysis Conference, Copenhagen,April 26-27, 2005.

[11] Mironov A., Mironovs D. Experimental application of OMA solutions on the model of industrial structure. IOP Conf. Ser.: Materials Science and Engineering, Vol 251, 2017.

[12] Piezo Film Sensors Technical Manual. Measurement Specialties, Inc. Norristown, PA, 1999. 